Revue internationale P.M.E.

Économie et gestion de la petite et moyenne entreprise

\title{
Le financement des PME par les banques : contraintes des firmes et limites
}

\section{Nadine Levratto}

Volume 3, numéro 2, 1990

URI : https://id.erudit.org/iderudit/1007977ar

DOI : https://doi.org/10.7202/1007977ar

Aller au sommaire du numéro

Éditeur(s)

Presses de l’Université du Québec

ISSN

0776-5436 (imprimé)

1918-9699 (numérique)

Découvrir la revue

Citer cet article

Levratto, N. (1990). Le financement des PME par les banques : contraintes des firmes et limites. Revue internationale P.M.E., 3(2), 193-213.

https://doi.org/10.7202/1007977ar
Résumé de l'article

Souvent considérées comme une priorité nationale en raison de leur contribution à la création d'emplois, les PMEfrançaises connaissent des difficultés financières chroniques. Le déséquilibre de leur structure de bilan n'est cependant pas attribuable aux seules spécificités endogènes de ces firmes ; il provient également des problèmes rencontrés par les PME pour établir des relations coopératives avec les banques. Le manque de fonds propres et les risques qui accompagnent un surendettement à court terme ne pourront en effet être résorbés que si les intermédiaires financiers acceptent de réviser leurs critères d'octroi du crédit qui désavantagent actuellement les unités de petites dimensions 


\title{
Le financement des PME par les banques: contraintes des firmes et limites de la coopération
}

\author{
Nadine LEVRATTO* \\ LATAPSES - CNRS
}

\begin{abstract}
RÉSUMÉ
Souvent considérées comme une priorité nationale en raison de leur contribution à la création d'emplois, les PME françaises connaissent des difficultés financières chroniques. Le déséquilibre de leur structure de bilan n'est cependant pas attribuable aux seules spécificités endogènes de ces firmes ; il provient également des problèmes rencontrés par les PME pour établir des relations coopératives avec les banques. Le manque de fonds propres et les risques qui accompagnent un surendettement à court terme ne pourront en effet être résorbés que si les intermédiaires financiers acceptent de réviser leurs critères d'octroi du crédit qui désavantagent actuellement les unités de petites dimensions
\end{abstract}

\section{ABSTRACT}

If small firms are often considered as a national priority because of their contribution to employment, it is impossible to neglect their financial difficulties which have often be pointed out. Nonetheless, their own behavior is not the only cause responsible of their balance sheet disequilibrium ; this observed fact is also due to the problems meet by small firms to build cooperative relationship with banks. In the french case, the lack of own funds, as well as the great risks induced by a disproportionate short-term indebteness, may be resorbed only if financial intermediaries accept to change the credit allocation criteria generally choosed. Owing to their increasing part in the realization of national product, nothing justify the fact that lenders still tend to favour big firms against small ones.

* Nadine Levratto est titulaire d'un D.E.A. d'économie de la production et achève actuellement son doctorat. Elle occupe un poste de boursière de doctorat pour ingénieur du C.N.R.S. au LATAPSES et est chargée d'enseignement à l'Université de Nice, France. Ses principaux centres de recherche concernent l'étude du fonctionnement du marché du crédit et le financement des entreprises. Adresse: LATAPSES, 1, rue A. Einstein, Sophia Antipolis, 06560, Valbonne, France. Premier manuscrit reçu: janvier 1989. 


\section{RESUMEN}

A menudo consideradas como una prioridad nacional por su contribucion a la creacion de empleos, pequenas empresas franceses encuentran dificultades financieras cronicas. $E$ desequilibrio de estrutura del balance, no solo se debea unas specifidades endogenas de estas firmes; proviene tambien de problemas encontrados por pequenas empresas para establecer relaciones de cooperacion con bancos. Lafaltta de fundos propios y los riesgos que acompanan el sobreadeudo a corto plazo podràn ser resueltos con tal que los intermediaros financieros acepten de revisar los criterios de concesion del credito que desventajan las unidades de pequena dimension. 


\section{Introduction}

Si les difficultés rencontrées par les PME. dans la mise en oeuvre de la production ou la réalisation d'un volume d'emploi prédéterminé sont fréquemment mises en exergue par les pouvoirs publics en France, l'accent sur l'étape du financement n'a été mis que récemment - encore que de manière discrète - dans le cadre du VIIème Plan. La prise en compte de cette question parmi les objectifs nationaux ne relève pas uniquement de considérations propres à la nécessaire survie de ces établissements, indépendamment du système économique, mais fait également intervenir des critères inhérents à la création d'emplois qu'elles peuvent engendrer (Greffe, 1984; Julien et Morel, 1986) et une contribution à l'expansion du système productif. En effet, leur insertion dans la concurrence internationale proposée par le VIIIe Plan repose sur l'hypothèse que les structures productives de petites et moyennes dimensions sont aptes à relayer les grandes entreprises parfois défaillantes en matière d'investissement, et donc d'impulser un certain dynamisme dans l'économie nationale (Marchesnay, 1982).

Il faut cependant mentionner que les diverses mesures d'aide mises en oeuvre en faveur du développement industriel et les multiples bonifications d'intérêt instaurées obéissent en général à une logique sectorielle, la taille des firmes ne constituant que très rarement un critère de choix. Or, dans le domaine de l'obtention des avantages financiers, les grandes entreprises se sont révélées mieux informées et plus aptes à surmonter les problèmes administratifs inhérents à la confection des dossiers que les PME, et surtout les TPE, ces dernières n'ayant donc pas toujours eu accès aux financements privilégiés dont elles pouvaient bénéficier (Baroin et Fracheboud, 1983).

Sans entrer ici dans le délicat débat relatif à la définition des caractéristiques économiques des entreprises petites et moyennes (Bernard et Ravix, 1988), il apparaît néanmoins nécessaire de dégager certaines de leurs spécificités susceptibles d'interférer dans les décisions des créanciers. Il faut tout d'abord mentionner le comportement spécifique des dirigeants d'unités de petite dimension qui tendent à opérer une confusion totale entre leur patrimoine et celui de l'entreprise, le corollaire de ce phénomène étant la réticence qu'ils manifestent à l'égard de l'entrée de capitaux extérieurs afin de préserver leur autonomie de gestion et de décision (Besse, 1983). Ce sentiment est encore renforcé par les exigences des banques qui, au moment d'accorder un crédit s'inquiètent de la présence de garanties personnelles, l'assimilation entre le dirigeant et l'entreprise étant ici manifeste (Dreher, 1980).

En second lieu, en dépit de leur similitude qui tient essentiellement au volume d'effectifs employés, l'ensemble des PME peut être segmenté en tenant compte de leur indépendance ou de leur insertion au sein d'un groupe industriel dont la surface financière est considérable ${ }^{1}$. Les unités de production s'inscrivant dans ce

1 Sur un plan purement pratique, une telle opération est rendue possible grâce aux enquêtes annuelles effectuées par l'INSEE sur les liaisons financières (Vassile, 1982). 
second cas de figure sont en effet avantagées dans leur recherche de fonds car elles disposent, du fait de leur situation, de garanties qui contribuent à sécuriser les bailleurs de fonds. De plus, les relations privilégiées que ces PME entretiennent avec d'autres entreprises, leurpermettentégalement de bénéficier de sources de financement alternatives.

Compte tenu de ces spécificités structurelles qui caractérisent l'ensemble des PME françaises, il s'avère possible d'opérer une distinction entre les différentes causes des problèmes de financement dont souffrent ces firmes, cette partition étant opérée grâce à la détermination de leur origine. La recherche des caractéristiques intrinsèques relevant de la taille ainsi que de la gestion des unités de petite et moyenne dimensions, et qui sont à l'origine d'une complexification de leurs possibilités de financement, fera donc l'objet d'une première partie, alors que la seconde sera consacrée à l'examen des interférences pouvant se produire dans les relations banques-PME.

\section{Les caractéristiques des PME qui entravent leur financement bancaire}

Les travaux se rattachant à la question du financement bancaire des PME et les enquêtes auprès de ces entreprises (Delatre, 1982; Vassile, 1982) font également état du caractère ténu du réseau de relations unissant les petites unités de production aux établissements bancaires et de l'insuffisante participation des PME aux circuits financiers. L'objet de cette première partie sera donc de montrer en quoi la gestion des entreprises petites et moyennes peut influencer leurs possibilités d'accès aux fonds prêtables, et, par conséquent, la réalisation de leurs projets d'investissement. $\mathrm{Si}$, depuis le début de la seconde moitié des années 1980, la croissance du chiffre d'affaires réalisé par les PMI est supérieure à celle de l'ensemble des entreprises françaises (Dessi, 1989), il faut cependant noter que ce redressement est consécutif à une longue période durant laquelle les performances de ces firmes furent médiocres (Delattre, 1986). En effet, comme l'indiquent les recherches réalisées dans le cadre de la Direction générale desétudes de la Banque de France (Larrieu et Lombard, 1982 et 1984; Laudy et Lombard, 1984; Delbreil et Laudy, 1987), depuis le début des années 1970 jusqu'en 1985, les PME (de même que les grandes entreprises industrielles) ont enregistré une certaine stagnation de leurs parts de marché allant de pair avec une augmentation relativement faible de leurs ressources propres 2 .

2 En effet, en raison du manque de fonds, ces entreprises sont contraintes à réviser à la baisse leurs programmes d'investissements, cette insuffisance de dynamisme pouvant poser des problèmes concernant l'obsolescence de leurs moyens de production. Le vieillissement de l'appareil productif risque ainsi de retentir sur les coûts de production, donc sur la compétitivité de ces firmes et leur insertion dans la concurrence internationale. 
Les solutions les plus fréquemment avancées dans le but de restaurer le dynamisme des PME-PMI font état du nécessaire assainissement de la structure de leurs bilans, la formation à la gestion des entrepreneurs concernés pouvant apparaître comme un préalable nécessaire à l'amélioration des ratios comptables. De même, l'acquisition d'une indépendance financière et l'amélioration des structures d'endettement semblent moins devoir passer par un développement des aides au renforcement des ressources propres que par l'élargissement de la diffusion des informations relatives aux subventions, déductions fiscales et procédures de financement privilégiées offertes par les différents organismes. Dans cette perspective, la simplification des modalités de crédit est censée apporter une solution au problème des obstacles administratifs rencontrés par les PME. Les questions de l'insuffisance des fonds propres, du déséquilibre de la structure de l'endettement et de la connaissance des opportunités permettant d'alléger le coût des prêts constitueront donc les trois axes autour desquels s'articulera l'analyse des spécificités des PME à l'origine des problèmes qu'elles rencontrent avec les banques.

\subsection{Les fonds propres}

Le manque de ressources propres dans les entreprises françaises est une constatation qui fait l'unanimité parmi les auteurs qui s'intéressent à l'analyse financière des firmes; ce problème étant ressenti avec une acuité particulière au niveau des structures de petite et moyenne dimensions, caractérisées par un risque de faillite plus élevé et une durée de vie plus brève que les firmes de plus de 200 salariés. Plusieurs raisons concourent à ce phénomène. Les principales résident dans la progression relativement faible du chiffre d'affaires sur les vingt dernières années, dans les spécificités des régimes fiscaux successivement en vigueur et dans le comportement patrimonial des dirigeants de PME, ces éléments ayant favorisé la diminution du pourcentage d'autofinancement dans la réalisation des programmes d'investissement (Delbreil et Laudy, 1987).

Le ralentissement de l'activité industrielle concommitant au premier choc pétrolier s'est traduit par une érosion généralisée de la croissance des recettes des entreprises. Pour l'essentiel, les PME ont davantage subi ce phénomène en raison de leur position particulière à l'égard de la demande; majoritairement dépendantes de la demande intérieure, ces dernières n'ont que peu profité de l'expansion des marchés étrangers parfois moins fortement touchés par la récession que l'économie française. En effet, alors que les PMI représentent $95 \%$ du total des entreprises et réalisent 40 $\%$ du chiffre d'affaires hors-taxe(Sessi, 1988), elles ne réalisent que 22,7\% du chiffre d'affaires des exportations de l'industrie française. Plus précisément, on peut dire que près de $60 \%$ des entreprises de moins de 500 salariés ne réalisent quasiment aucune exportation; seule une infime minorité, située dans le sous-groupe des PME employant entre 200 et 500 salariés, a exporté en, 1986, plus de la moitié de leur chiffre d'affaires (ibid.). 
Le recul des recettes réalisées grâce aux exportations, caractéristique de la période 1982-1986, explique en partie pourquoi, en dépit des rémunérations plus faibles dans les PMI, ces dernières présentent un taux de marge inférieur à celui des firmes de plus de 500 salariés. C'est ainsi que ce taux, appréhendé par le rapport entre l'excédent brut d'exploitation exprimé en pourcentage et la valeur ajoutée, est respectivement égal à 21,9 et $24,2 \%$ pour l'année 1986 (Sessi, 1988). Mais d'autres indicateurs comptables permettent de rendre compte de la fragilité financière des PME. L'examen du rapport Mayoux (1979) permet de mettre en lumière l'évolution des disparités entre les PMI et les grandes entreprises en ce qui concerne l'évolution des passifs et des fonds propres. Si en 1967 les PMI faisaient autant appel à l'endettement qu'à leurs fonds propres pour satisfaire leurs besoins de financement, cette situation n'a cessé de se dégrader, puisqu'en 1976l'endettement en représentait les deux tiers, pour se stabiliser, depuis 1985, aux alentours de $60 \%$.

L'inertie des performances financières s'est ainsi trouvée à l'origine d'une réduction des marges bénéficiaires et donc d'une diminution des possibilités de réinvestissements de ces fonds dans l'entreprise, ce phénomène ayant encore accentué le déséquilibre de la structure financière des PME au détriment des postes de haut de bilan.

Par ailleurs et durant de nombreuses années, les dispositions figurant dans le droit fiscal ont provoqué une distorsion dans le coût du capital en favorisant le financement par dette par rapportà la constitution de fonds propres (Borel et Bouvier, 1985). En effet, la croissance de ces derniers par dotation des réserves suppose la mise en évidence d'un bénéfice imposable qui risque de pénaliser l'entreprise, alors que les charges découlant d'un surcroît d'endettement sont supportées par le compte d'exploitation (Hourcade et Rosenberg, 1980). De même, l'émission d'actions nouvelles en vue d'augmenter le capital de la firme est accompagnée d'un coût fiscal dissuasif pour l'entrepreneur. Toutefois, les mesures fiscales prises, notamment pour favoriser l'actionnariat, visent à rétablir une certaine neutralité grâce à la possibilité de déduire du bénéfice imposable $50 \%$ des dividendes alloués aux actions ou parts représentatives des apports en numéraire, ce qui, couplé au principe du compte d'épargne en actions, peut créer une réelle synergie au niveau du financement des PME. De par son caractère incitatif, la fiscalité peut en effet contribuer à modifier les comportements des dirigeants d'entreprises en faveur de la constitution des ressources propres, ce changement d'attitude pouvant résoudre, au moins partiellement, le problème de la sous-capitalisation des PME qui obère parfois leur survie (Baroin et Fracheboud, 1983).

Enfin, la faiblesse des mises en réserve ne provient pas exclusivement de l'insuffisance des excédents d'exploitation, mais également du comportement patrimonial des dirigeants de PME (notamment de moins de 50 salariés) qui craignent de perdre une partie de leur pouvoir de gestion et de décision en introduisant de nouveaux actionnaires. Conformément aux thèses avancées dans le cadre de la théorie de l'agence (Charreaux, 1987; Stiglitz, 1985) qui fait état des problèmes liés 
à l'exercice du pouvoir dans des firmes dont le capital est détenu par des actionnaires de différente puissance et où les banques interviennent par le biais de l'octroi de crédit, les dirigeants d'unités petites et moyennes se trouvent souvent confrontés à la question de l'évaluation du caractère bénéfique de l'entrée de partenaires. En effet, que ces derniers désirent ou non prendre une part active à la gestion de la production de la PME dans laquelle ils investissent, tous désirent avoir un droit de regard sur les stratégies de croissance et de développement choisies. Si cette attitude s'explique par la recherche d'une rémunération optimale de l'épargne sous la forme de dividendes, elle peut également être perçue comme une forme d'ingérence propice à un recul de l'indépendance du propriétaire initial. Il apparaît d'ailleurs nécessaire de mentionner ici que le caractère familial de la plupart des unités de petite dimension constitue une entrave au développement de l'actionnariat dans ces sociétés, le problème des fonds propres étant ressenti comme une affaire personnelle, dépendant exclusivement du chef d'entreprise. Dans ce domaine, l'intervention et les conseils des prêteurs peuvent se révéler précieux, puisque par le biais des ratios prudentiels, ces partenaires peuvent exercer des pressions visant à faire respecter le principe fondamental selon lequel, pour être libre, l'entreprise doit travailler avec son propre argent. L'arbitrage systématique en faveur de fonds extérieurs implique au contraire des contraintes financières importantes (de Wulf et Percie du Sert, 1983), l'existence d'un lourd service de la dette pouvant se révéler nuisible à la croissance de la firme aussi bien qu'à la mise en place de processus de diversification ou de reconversion.

En définitive, l'insuffisance des ressources propres constitue un problème vivement ressenti par les petits entrepreneurs, car elle les empêche d'avoir recours à l'autofinancement qui est pourtant très valorisé par les dirigeants de $P M E$. Il s'avère donc nécessaire d'inciter ces derniers à faire davantage appel à l'épargne afin de restaurer l'image de marque de la firme auprès de ses partenaires, l'équilibre entre endettement et fonds propres qui caractérisait le financement des PMI dans les années 1960 n'ayant cessé de se détériorer (Mayoux, 1979). La banalisation du capitalrisque découlant d'un développement de l'interface entre les entrepreneurs et les épargnants proches géographiquement, tout comme laconsolidation de l'endettement bancaire à court terme en fonds propres qui relève du même principe de participation directe, peut se révéler financièrement rentable pour les entrepreneurs et leurs partenaires financiers; mais l'instauration de telles pratiques nécessite une modification de la réglementation et des comportements des agents concernés. A plus court terme, la mise en place des prêts participatifs qui, bien que remboursables, figurent parmi les capitaux propres dans le bilan des entreprises bénéficiaires, constitue une solution satisfaisante, car elle permet d'accompagner la croissance des PME performantes, mais temporairement bloquées dans leurs perspectives d'expansion par une capacité d'endettement limitée (Levratto et Torre,1986).

\subsection{La structure de l'endettement}

La taille des entreprises constitue un facteur discriminant en matière de structure d'endettement, car elle conditionne leurs possibilités d'accès aux diverses 
sources de fonds, le nombre d'opportunités de financement offertes augmentant parallèlement à la taille des firmes considérées. Les obstacles rencontrés par les PME dans leurs demandes de crédits sont généralement mentionnés dans les études effectuées au niveau européen ${ }^{3}$ qui font état des difficultés rencontrées pour couvrir les besoins en capitaux, d'autant que les emprunts bancaires constituent quasiment la seule modalité de satisfaction des besoins de financement. Il apparaît en effet, que l'intermédiation bancaire occupe une place importante dans la structure d'endettement des PME (Baroin et Fracheboud, 1983). Ces dernières ont des difficultés à s'introduire sur le marché obligataire et, a fortiori, sur les marchés internationaux des capitaux alors que les grandes entreprises peuvent y être présentes, leur taille leur permettant en outre de bénéficier de crédits partiellement ou totalement désencadrés (Laudy et Lombard, 1984).

Au-delà de cette prépondérance de l'endettement bancaire parmi l'ensemble des ressources dont disposent les petites unités de production, il apparaît important de mentionner la prééminence des crédits à court terme qui représentent plus de la moitié du total des dettes contractées par ces entreprises. Il s'avère toutefois ici nécessaire de distinguer les PME indépendantes de celles qui appartiennent à des groupes, car la structure de bilan de ces dernières tend à se rapprocher de celle des grandes entreprises «... par la partélevée de l'endettement long et le poids relativement faible des bas de bilan» (Vassile, 1982). En dépit de certaines fluctuations conjoncturelles, les plus petites entreprises connaissent en effet des besoins de trésorerie croissants, la couverture des besoins en fonds de roulement d'exploitation par des ressources stables étant un résultat difficile à obtenir d'autant que les PME craignent d'être liées à long terme. En raison de ce décalage, ces firmes sont obligées de solliciter un important volume de crédits à court terme auprès des banques qui leur accordent l'essentiel des découverts et des crédits de trésorerie qu'elles réclament. Ainsi, non seulement elles se financent mal, mais en outre, elles choisissent des modes de financement onéreux.

Le renouvellement quasi-systématique de ces avances et leur progression dans les bilans des PME rend ces dernières largement tributaires des établissements bancaires et des modifications affectant les critères ou les procédures d'octroi du crédit. Cette situation défavorable à l'élaboration d'une stratégie de croissance autonome provient également de la faiblesse des crédits inter-entreprises dont bénéficient les PME, leur pouvoir économique étant insuffisant pour inciter leurs foumisseurs à leur faire crédit alors qu'en revanche elles sont souvent sollicitées par leurs gros clients pour différer des encaissements. Cette position par rapport aux

3 Baroin et P. Fracheboud (1983) font référence au Rapport Kolbenschlag sur «la lutte contre le chômage par l'encouragement des petites et moyennes entreprises dans la Communauté européenne», Comité économique et social, Bruxelles, 1981. Cette étude démontre que les besoins en capitaux des PME sont en augmentation constante alors que les possibilités d'obtenir des fonds croissent dans une moindre mesure, ce qui crée des problèmes d'ajustements financiers et de gestion. 
bailleurs de fonds explique pourquoi $80 \%$ des PME françaises se sentent dépendantes des banques (Crédit Hôtelier, 1980).

\subsection{L'accès à l'information}

La diversité des modes de financement privilégiés a fait l'objet de nombreuses critiques car la multiplication des formules de prêt proposées par les établissements bancaires et non bancaires constitue un handicap pour les dirigeants de petites unités de production qui peuvent avoir du mal à choisir la modalité de financement la mieux adaptée au projet d'investissement qu' ils envisagent de réaliser. ${ }^{4}$. Toutefois, l'existence de modes de financement spécifiques a été motivée par la nécessité d'impulser une dynamique de croissance des PME et plus généralement de l'industrie, la crise se trouvant à l'origine de la mise en application successive de différents prêts aidéss. Par ailleurs, pour répondre au même type de besoins, les Pouvoirs Publics ont mis en place le CEPME (Crédit d'équipement des petites et moyennes entreprises), établissement financier non bancaire, dont le rôle est d'allouer des fonds aux petites entreprises dynamiques cherchant à investir soit pour moderniser leurs techniques de production, soit pour pénétrer de nouveaux marchés. La simplification du système de crédit en vigueur était fréquemment demandée par les petits entre-

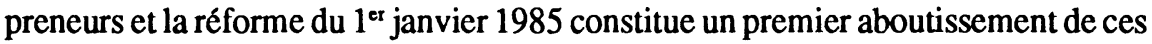
requêtes, puisqu'elle a conduit à déterminer une enveloppe de crédits privilégiés unique devant favoriser le renouvellement du tissu industriel, l'effort d'investissement et l'exportation (Notes Bleues, 1985)). Notons également que la réforme récente des prêts à taux bonifiés qui consacre le désengagement de l'État dans ce domaine accorde toutefois une large priorité aux unités de petite taille, puisque ces dernières continuent à bénéficier de financement à des taux inférieurs à celui du marché.

Toutefois, pour être efficace, l'effort de simplification des procédures doit s'accompagner d'une diffusion des informations assurée par les banquiers qui, a priori, ont intérêt à ce que leurs débiteurs soient dans la meilleure situation financière possible (Levratto et Torre, 1986). Les bailleurs de fonds doivent donc devenir les interlocuteurs privilégiés des dirigeants de PME, cette coopération ne devant pas uniquement concerner les prêts accordés à l'entreprise mais également la gestion

4 Stiglitz (1985) qui considère que la taille des entreprises constitue un élément discriminant quant à leurs possibilités d'accès à l'information, ce phénomène pouvant entraîner des répercussions importantes au niveau de la réalisation des projets d'investissement. En effet, les firmes ayant la meilleure connaissance des modalités de financement peuvent bénéficier des prêts aidés qui ne sont pas forcément connus de leurs concurrents, les coûts des crédits se révélant très différents selon les procédures suivies.

5 En 1984, les prêts à taux bonifiés ont fait l'objet d'une réforme qui a remplacé les six catégories en place par une seule procédure connue sous le nom de Prêts Spéciaux à l'Investissement (P.S.I.), dont le coût se situe en deça du taux du marché. Par ailleurs, les prêts qui ne bénéficient d'aucune aide budgétaire ont vu baisser le taux qui leur était assorti en raison de la diminution du taux de base bancaire. 
générale de la firme. En effet, sans intervenir directement, les banquiers pourraient jouer des rôles de conseillers et inciter les entrepreneurs à assainir, si besoin est, la situation comptable et financière de leur unité. L'interface banque-entreprise prendrait ainsi une nouvelle dimension qui permettrait de dépasser les contradictions entre prêteurs et emprunteurs.

Si les relations existant entre ces deux pôles sont souvent antagoniques, c'est en raison de la nature exclusivement financière des flux qui lient ces deux entités économiques; il apparaît donc qu'en développant les rapports entre ces agents et en leur donnant conscience du caractère commun de leurs intérêts, le travail de chacun sera facilité. Ainsi, les lacunes que présentent les entrepreneurs dans le domaine de la gestion courante peuvent être comblées par l'intervention de financiers experts dans ce domaine qui, en prodiguant des conseils pourront améliorer la rentabilité de la firme emprunteuse et donc réduire les risques encourus au moment du prêt. Le caractère complexe du produit «crédit» ou «avance de fonds» explique ainsi le succès rencontré par les Organismes de capital risque (OCR) qui, outre la distribution de ressources aux entreprises demandeuses, assurent une assistance technique pouvant concerner non seulement le processus de production et la distribution des produits, mais également la gestion même de l'entreprise (Geoffron, 1990). Il va de soi que cette coopération n'est pas neutre dans la mesure où l'essentiel des PME faisant appel aux OCR sont rachetées par ces derniers au terme du contrat financier.

La multiplicité des types d'avances de fonds s'adressant aux PME ainsi que le grand nombre d'établissements financiers bancaires ou non bancaires travaillant sur ce segment de marché permet de considérer que le financement de la petite entreprise ne bute pas sur une insuffisance des ressources disponibles. Les obstacles à la distribution de crédits relèvent plutôt du comportement des dirigeants des firmes et des préjugés des éventuels apporteurs de fonds (Mignot, 1979). Ces deux groupes d'agents se caractérisent trop fréquemment par une méfiance réciproque et tendent à souvent négliger les points communs existant entre les objectifs respectivement visés. Cependant si une nécessaire évolution des relations banques-entreprises ne peut se réaliser qu'à l'aide de réformes des modes de financement, elle requiert également une modification des structures comportementales des agents concernés, si bien que la prise en considération de cet impératif apparaît comme une étape préalable à tout changement.

En définitive, les difficultés d'accès aux fonds prêtables rencontrées par les PME ont des causes multiples pouvant être scindées en deux grandes catégories. La première relève de la gestion des firmes concernées qui, suivant une approche en termes de cycle de vie, se trouvent dans une situation de précarité financière consécutive aux phases de création et de lancement. Cette période d'essoufflement, marquée par une fragilité financière de la firme, se traduit par une faiblesse des fonds propres, un taux d'autofinancement insuffisant et une structure d'endettement déséquilibrée, tous ces éléments nuisant à la perpétuation du développement de l'entreprise. Le second groupe de facteurs responsables des difficultés de croissance 
rencontrées par les PME fait davantage référence au comportement de leurs dirigeants. Ces derniers souffrent en effet d'une formation insuffisante qui, ajoutée à l'insuffisance de l'encadrement et à la multiplication des tâches à assumer, explique leur incapacité à recueillir les informations concernant les prêts ou les aides dont ils pourraient tirer parti. Ce constat permet de supposer que l'amélioration des conditions financières des unités de petite dimension passe parune meilleure compréhension de la rationalité financière et bancaire del'environnement auquel elles sontconfrontées, l'acquisition d'une logique plus conforme à celle des bailleurs de fonds nécessitant l'abandon du comportement patrimonial encore trop souvent adopté par les «patrons» de PME, ce qui nuit à la véritable intégration de ces firmes dans le système productif et les circuits financiers nationaux.

\section{La question des relations banques-PME}

La dégradation régulière des taux d'autofinancement a contribué à accroître le financement externe, car, en dépit du blocage de l'investissement industriel (Artus et Muet, 1982), la formation brute de capital fixe connaît une croissance régulière venant alourdir les besoins de financement de l'ensemble des entreprises françaises. Si l'augmentation de l'endettement touche essentiellement les grandes entreprises, les PME dont les ressources propres s'amenuisent, connaissent également une «situation de financement forcé» (de Wulf et Percie Du Sert 1983; Delbreil et Laudy, 1987) où l'augmentation des concours bancaires sert essentiellement à retarder les cessations de paiement et à effectuer les investissements nécessaires au strict renouvellement des biens capitaux défaillants. Il est en effet frappant de constater que les investissementsréalisés par les PMI sont peu fréquemment destinésàl'amélioration de la productivité (Devilliers, 1987), mais servent essentiellement à renouveler les capacités de production. A terme, la détérioration des comptes des entreprises et l'instauration de ce type de palliatifs risque de nuire à la mise en place d'une véritable stratégie de croissance, les décideurs étant exclusivement préoccupés par des questions financières immédiates, alors que les épargnants-investisseurs potentiels, qui jugent les placements dans l'industrie trop aléatoires, risquent d'opter en faveur d'emplois plus sûrs et plus rentables.

La création «...d'un climat général de confiance pour l'entrepreneur à l'intérieur d'un cadre financier, fiscal, social et économique stable...» (Baroin et Fracheboud, 1983) apparaît comme un objectif fondamental pour les Pouvoirs Publics, la dépendance excessive des PME vis-à-vis des banques ne pouvant constituer un fondement solide pour le développement industriel. Toutefois, plus que d'une restriction draconienne du volume de crédit dont elles peuvent bénéficier, les petites unités ont besoin de restructurer leur endettement global au détriment du court terme afin de stabiliser leur situation financière. Une modification de leurs conditions d'accès au crédit apparaît donc nécessaire à l'insertion véritable des petites firmes (surtout indépendantes) dans la concurrence nationale et internationale, carl'obtention 
de fonds se révèle indispensable àl'acquisition de moyens de production performants pour fabriquer des produits compétitifs.

\subsection{Le problème général de l'accès aux fonds}

En dépit des mesures adoptées en vue de faciliter l'accès au crédit pour les PME (prêts bonifiés spécifiques, prêts participatifs renforcés...), le transfert de l'épargne vers ces agents s'avère être une opération délicate en raison de la surévaluation des risques par les investisseurs en bourse. La méfiance de cette catégorie de bailleurs de fonds est d'ailleurs justifiée par le fait que les PMI sont plutôt situées dans des secteurs en récession. En effet, les entreprises de moins de 500 salariés détiennent la majorité des parts de marché dans les secteurs dont la croissance est comprise entre - 5 et 2,5\% sur la période 1979-1982 (Amar, 1987). A l'inverse, sur les créneaux les plus dynamiques, cette catégorie d'entreprises ne bénéficie d'aucun avantage, leurs performances appréhendées par le taux de marge et le taux de rentabilité financière se révélant légèrement inférieures à celles des grandes entreprises. Il existe toutefois une catégorie d'agents financiers, spécialisés dans la capital-risque (Geoffron, 1987), dont le rôle est de diriger les ressources des individus excédentaires vers les unités déficitaires et qui, par ce biais, réalisent une «transformation» de l'épargne liquide en financement à long terme (Vatteville, 1972). De même, l'accès indirect au marché international des capitaux a été ouvert aux PME grâce à certains établissements financiers qui ont souscrit des emprunts à l'étranger afin d'accorder des prêts en francs à des entreprises nationales (Berger, 1986). En outre, le développement du second marché et des bourses de province, ainsi que l'ouverture du marché obligataire à des firmes de moyenne dimension, a permis à leurs dirigeants de rencontrer des offreurs de capitaux propres et donc de pouvoir bénéficier d'une épargne qui jusqu'alors leur échappait.

La régionalisation de la politique des banques apparait également comme un élément prépondérant dans le rapprochement entre les bailleurs de fonds et les PME (Salvadori, 1986), le processus de spécialisation dans lequel s'engagent les premiers les conduisant à resserrer les liens qu'ils entretiennent avec des entreprises absentes du marché des capitaux. La mise en place de la dérégulation et de la déréglementation du système financier peut ainsi conduire les établissements bancaires à la recherche de nouveaux réseaux de clients (Pastre 1985), les unités de petites et moyennes dimensions constituant une cible privilégiée en raison de la fréquence élevée de leurs appels au crédit. Comme les entreprises subissent un rationnement quantitatif au niveau des fonds prêtables ou des capitaux demandés sur les différents segments du marché monétaire et financier, le report sur le marché des crédits bancaires constitue pour elles une stratégie fréquemment retenue. La demande de crédit des firmes de moins de 500 salariés pouvant compenser la chute du montant de crédits bancaires alloué aux grandes unités en raison du phénomène de titrisation, nombreux sont les établissements financiers cherchant à fidéliser, voire à conquérir, la clientèle des PME. A titre d'exemple, on peut citer la démarche du Crédit Agricole qui, jusqu'à 
récemment spécialisé dans le financement des activités agro-alimentaires, a décidé d'élargir son champ d'action aux entreprises petites et moyennes.

\subsection{Les critères d'attribution du crédit}

Lorsqu'ils sont confrontés à une demande de crédit, les prêteurs examinent le dossier à la lumière de trois catégories de critères faisant intervenir des éléments représentatifs de différents aspects de l'emprunteur potentiel (Portait et Noubel, 1982):

- la recevabilité, proche du droit, permet de juger de la légalité de la requête;

- les aspects financiers aident le prêteur à évaluer les risques pris, grâce au calcul de ratios comptables qui limitent le volume de l'endettement par rapport aux fonds propres, de même que le montant des remboursements futurs en fonction de la marge brute d'autofinancement prévisionnelle;

- les objectifs économiques concernent la rentabilité anticipée du projet d'investissement et son adéquation avec les impératifs nationaux.

La rigidité de cette grille de décision traditionnelle fait parfois l'objet de critiques, dont les plus fortes portent sur la définition des ratios financiers qui interdisent toute prise en considération des particularités propresà certaines activités, cette insuffisance de souplesse conduisant parfois à la non-application de certaines contraintes. Le respect de l'ensemble de ces éléments constitue en outre une limitation aux possibilités d'endettement des entreprises, la grande masse des PMEPMI se trouvant ainsi fréquemment pénalisée, même si les établissements financiers spécialisés auxquels elles s'adressent tendent à assouplir ces contraintes, notamment en contrepartie du paiement d'intérêts plus élevés.

Toutefois, en ce qui concerne l'octroi de crédits d'extension et de fonctionnement et donc dans le cas du financement de PME déjà existantes, la variable de décision la plus représentative pour les institutions financières demeure la position de marché occupée par la firme emprunteuse. La situation de cette dernière, eu égard à la concurrence et sa capacité d'attirer et de fidéliser la clientèle, traduit en effet son dynamisme et sa compétitivité (Levratto et Torre, 1986). Les décideurs bancaires seront particulièrement sensibles à ces aspects dynamiques qui, ajoutés à l'analyse des indicateurs comptables, permettent d'évaluer les capacités de remboursement du débiteur. Si la part de marché accaparée par une PME se révèle déterminante par les bailleurs de fonds, c'est conformément au principe des barrières à la mobilité mis en évidence par R. Caves et M. Porter (1977). Fondée sur l'idée de frein à la mobilité intersectorielle des entreprises, la notion de barrière à la mobilité traite de l'accès à l'intérieur d'un secteur d'un groupe de firme à un autre (Rainelli, 1989), les groupes de firmes étant définis à partir de caractéristiques structurelles et segmentant le secteur (Mascarenhas et Aaker, 1989). Ainsi, les barrières à la mobilité protègent «les firmes appartenant à un groupe à la fois contre l'entrée d'une firme du même secteur appartenant à un autre groupe» (Rainelli, 1989). C'est donc sur ce principe qu'est 
élaborée la thèse suivant laquelle les firmes de moins de cinq cents salariés exercent en général leur activité sur des marchés géographiquement bien délimités (régionaux ou locaux) et, pour cette raison, n'entrent en concurrence qu'avec des unités de production de taille équivalente et situées dans leur environnement immédiat (Caves et Porter, 1977). Il faut toutefois souligner, à l'instar de B. Guesnier (1986), que les PME constituent une population au profil extrêmement changeant, le renouvellement des entreprises de moins de 500 salariés s'effectuant à un rythme élevé. Le caractère localisé des marchés sur lesquels elles interviennent demeure néanmoins confirmé par la relation directe existant entre la taille de l'entreprise et le taux d'exportation du chiffre d'affaires (33,9\% pour les entreprises de 500 salariés et plus, $18,7 \%$ pour les PMI de 100 à 199 salariés et $9,7 \%$ pour les petites entreprises industrielles employant entre 20 et 49 salariés (Sessi, 1988).

Notons cependant que cette partition des marchés se trouve atténuée par la prise en considération de la flexibilité définie par G. Stigler (1939) comme étant l'apanage des petites entreprises. Leur capacité d'adaptation due à la spécificité des technologies qu'elles utilisent permet ainsi aux PME de concurrencer avec succès des firmes de taille plus importante qui, en dépit de la réalisation d'économies d'échelle, subissent des coûts moyens élevés (Mills et Schumann, 1985). Il faut en particulier mentionner que les coûts salariaux supportés par les PME françaises sont $20 \%$ inférieurs à ceux des grandes entreprises et que, toujours dans le domaine des relations avec le personnel, les conventions collectives sont moins élaborées dans les petites et moyennes unités que dans les grandes (on peut à titre d'exemple citer les cas du paiement du complément de salaire versé lors des congés maternité dans les grandes firmes, la réduction du nombre d'heures de travail pour les salariés membres des comités d'entreprise ou des cercles de qualité...). Néanmoins, les caractéristiques de l'emprunteur au sein du marché sur lequel il opère, et la place qu'il y occupe, demeurent déterminantes dans l'octroi de crédit, si bien que les entreprises financées en priorité sont celles qui possèdent les débouchés les plus importants, contrairement aux principes développés dans les analyses d'inspiration marshallienne ou walrasienne ${ }^{6}$. Or, même si l'on considère au départ une symétrie du marché étudié, l'introduction d'une discrimination au niveau du coût du crédit conduit à la réalisation d'un équilibre asymétrique (Flaherty, 1980) favorable aux entreprises bénéficiant des meilleures conditions de financement, c'est-à-dire des taux les plus faibles.

6 L'existence de marchés imparfaits et de nouvelles formes de concurrence (Jacquemin, 1985) conduit en particulier à l'érection de barrières à l'entrée prenant la forme de coûts irrécupérables. Ces derniers reposent sur des dépenses d'investissement incompressibles et indispensables à la pénétration sur le marché visé (recherche-développement, publicité...), la réalisation de ces programmes alourdissant les charges inhérentes àl'installation de l'entreprise et faisant de ce fait peser des contraintes encore plus strictes sur leur rentabilité. L'entrée de nouveaux concurrents devient ainsi très limitée, car les banques refusent fréquemment de leur accorder les crédits nécessaires en raison de l'incertitude qui tend à réduire leurs chances de succès. 
La rentabilité des PME emprunteuses constitue également un critère important pour les prêteurs, la réalisation d'un chiffre d'affaires élevé permettant de minimiser les risques de faillite, et donc d'augmenter les chances de remboursement. Le calcul des différents indicateurs (Camus, 1981; Levratto, 1988a) indique que les PME indépendantes ont une rentabilité supérieure à celle des unités appartenantà des groupes et aux grandes entreprises, ce constat général devant toutefois être affiné par la prise en compte des divisions sectorielles. En effet, si les PME réalisent dans l'ensemble des niveaux d' investissement qui suivent une tendance décroissante entre 1979 et 1987 et présentent une structure des emplois moins immobilisée que celle des grandes entreprises, d'importantes disparités sectorielles doivent être mentionnées. C'est ainsi que les performances réalisées dans les PME situées dans l'industrie et le commerce de gros se révèlent sensiblement meilleures que celles caractérisant les entreprises de moins de 500 salariés appartenant au commerce de détail et au bâtiment, génie civil et agricole. ${ }^{7}$ Il reste cependant possible de considérer que la bonne rentabilité d'ensemble caractéristique des PME indépendantes provient dans une large mesure de la relative faiblesse de leur intensité capitalistique mesurée par le ratio immobilisations d'exploitation/effectifs, mais trouve également son origine dans la disparition, pendant la crise, des PME les moins rentables (Vassile, 1982). C'est ainsi que conformément aux approches en termes de sélection naturelle qui supposent, entre autres, l'élimination des unités utilisant des méthodes de production inefficientes (Baumol, 1982), seules les entreprises les mieux adaptées aux contraintes imposées par les fournisseurs et aux exigences manifestées par les clients ont pu passer le cap des difficultés provoquées par les deux chocs pétroliers et la crise du milieu des années 1980.

Toutefois, en dépit des bons résultats réalisés par les PME dont la position s'est trouvée encore renforcée à la suite de la reprise boursière amorcée à la fin du premier semestre 1988, les banques tendent à surestimer les risques encourus en finançant ce type de firmes et surtout celles nouvellement créées, l'importance des garanties personnelles demandées aux dirigeants constituant un instrument de rationnement fréquemment employé (Baroin et Fracheboud, 1983). Les relations de clientèle jouent aussi un rôle fondamental dans l'octroi de prêts aux PME, la confiance accordée par le prêteur au producteur pouvant apparaître comme un substitut à l'analyse financière, notamment en raison du caractère souvent peu formalisé des documents comptables présentés par les dirigeants d'unités de petite dimension. L'instauration de liens entre les deux parties se révèle donc une étape importante de toute procédure de financement dans la mesure où les conditions des crédits contractés dépendent en grande partie du "pouvoir de marchandage» exercé par l'emprunteur sur le bailleur de fonds (Gourlaouen, 1978; Levratto, 1988b). Toutefois, les jeunes créateurs d'entreprise se trouvent ici dans une situation défavorable en raison de leur méconnaissance des cadres bancaires et de la rationalité

7 Les données chiffrées figurent dans une étude réalisée dans le cadre de la Banque de France par Delbreil et Laudy (1987). 
spécifique des établissements auxquels ils s'adressent, le développement d'une assistance technique pouvant constituer une mesure à adopter afin de faciliter l'accès des PME aux fonds prêtables.

\subsection{Le coût de l'accès au crédit}

Les études entreprises sur les conditions de financement offertes aux $\mathrm{PME}^{8}$ font état d'une discrimination venant les pénaliser et susceptible d'entraver leurs stratégies de développement en leur imposant des coûts plus élevés que ceux qui prévalent pour les firmes de dimension plus importante. La thèse la plus répandue est en effet celle mise en évidence par J.K. Galbraith (1959) qui considère que les petites unités de vente et de production sont financièrement étranglées et victimes du comportement restrictif des banques dans leurs demandes d'emprunts, des inégalités flagrantes apparaissant entre le secteur oligopolistique, peu concerné par le gel des crédits et les PME exposées aux aléas du marché.

Les coûts des crédits contractés se révèlent en effet très différents selon la taille de la firme emprunteuse. Cette situation peut se justifier, car il existe en moyenne plus de risque à prêter à une petite entreprise (Vatteville, 1972), comme l'indiquent les taux de faillite très élevés dans ce groupe'. Toutefois, la généralisation de ce type de procédure peut apparaître comme un élément dissuasif pour certaines PME, car les frais financiers élevés et les garanties personnelles demandées au dirigeant peuvent être à l'origine d'une confusion néfaste entre les avantages de l'individu et ceux de la société. Les enquêtes sur le coût du crédit aux entreprises réalisées par la Banque de France (cf. la partie statistique du Bulletin Trimestriel) montrent en effet que les charges d'intérêts sont inversement liées au montant de l'avance accordée non seulement pour des raisons tenant à la réalisation d'un montant de bénéfice jugé minimal par la banque, mais aussi pour des motifs liés au profil du débiteur. Les petits crédits étant la plupart du temps demandés par des entreprises possédant une surface financière modeste, le bailleur de fonds est souvent enclin à réagir en intensifiant ses exigences, négligeant par là même les effets pervers produits par ce type de stratégie ${ }^{10}$.

Les taux d'intérêts apparents mesurés par le rapport frais financiers/total des dettes sont ainsi plus élevés dans les petites entreprises que dans les grandes en raison de la part élevée des modalités de financement privilégiées (et notamment des crédits

8 En effet, les firmes industrielles, exceptées celles qui appartiennent au secteur de la viande et des produits laitiers, se révèlent plus rentables que les entreprises de services et notamment pour les services aux entreprises et les transports (Vassile, 1982).

9 Cette caractéristique se retrouve tout au long des étapes de la croissance des PME, le coût du crédit diminuant avec l'âge de l'entreprise. En effet, le risque décroît si la qualité de la gestion s'améliore, l'expérience et la spécialisation de la firme jouant ici un rôle considérable (Gupta, 1969).

10 Pour une analyse de la sélection adverse produite par l'augmentation du taux d'intérêt ou du nantissement du crédit (Stiglitz, 1981). 
à l'exportation) dans l'endettement global de ces dernières (Vassile, 1972; Delbreil et Laudy, 1987), bien que, compte tenu de leur sous-bancarisation, les PME subissent des frais financiers globaux inférieurs à ceux des grandes unités. La mise en oeuvre d'actions visant à faire bénéficier les petites unités d'emprunts à taux réduits dans le cadre du CEPME, des Sociétés de développement régional ou encore de la SOFARIS, ainsi que le renforcement du plafonnement des taux pratiqués dans le cadre des Prêts spéciaux à l'investissement débloqués par les banques ou le réseau mutualiste ont comme objectif de faciliter le financement des PME tout en contrôlant son coût. Le succès de ces mesures n'a toutefois été que partiel, car si les distinctions sectorielles ont toujours été exprimées de manière explicite dans les conditions à remplir, il n'en a pas toujours été de même pour la taille, ce qui explique pourquoi la majeure partie de ces enveloppes spécifiques a été dirigée vers les grandes entreprises.

L'application du principe d'encadrement du crédit a également amputé les possibilités d'emprunt des PME qui ont supporté l'essentiel des contraintes et des restrictions impliquées par ce type de politique monétaire. En effet, de 1976 à 1983, la conjoncture économique n'a pas permis aux entreprises de réaliser des taux d'autofinancement leur permettant de mettre en oeuvre leurs projets d'investissement, à cette réduction des performances propres étant venue s'ajouter la diminution de l'offre de crédits. Les petites unités de production ont été les plus touchées par le contrôle de l'offre de crédits, puisque durant les périodes de véritable «morsure»" ce sont essentiellement les PME qui ont été rationnées comme l'indique la baisse de leurs emplois et de leurs besoins en fonds de roulement (Laudy et Lombard, 1984; Sessi, 1988). En effet, le besoin de financement externe des grandes sociétés a augmenté alors que l'encadrement du crédit pesait plus lourdement sur les systèmes bancaire et productif. La demande des grandes sociétés et des PME les plus importantes a donc été satisfaite grâce à l'intervention des prêteurs non-bancaires et à la multiplication des crédits de trésorerie. En revanche, l'allègement des besoins d'exploitation des plus petites firmes est allé de pair avec une croissance moindre et un volume d'endettement inférieur.

Il apparaît ici que l'insertion des PME dans le crédit inter-entreprise constitue un élément tendant également à précariser leur situation financière. En effet, les petites entreprises accordent en général des avances à leurs clients les plus importants, si bien qu'aux charges financières inhérentes à la détention de créances à rendement nul viennent s'ajouter les risques liés à ces mêmes créances. Ce phénomène permet d'expliquer en partie l'importance des emprunts de trésorerie effectués par les unités de petite dimension, et contribue également à accroître l'estimation des risques encourus par les banques qui sont confrontées à des demandes de fonds émanant de ces entreprises.

11 On entend par morsure de l'encadrement du crédit les conséquences effectives de la restriction monétaire sur la demande de crédit. 
Le caractère excessif de la dépendance des petites entreprises vis-à-vis des banques ne contribue donc pas à améliorer les relations entre ces deux pôles économiques, ces unités de productions étant les premières à ressentir les contractions de l'offre de crédits. L'importance des intérêts en jeu constitue l'une des causes de ce phénomène, puisque les répercussions sur l'emploi de la liquidation d'une entreprise nationale sont plus graves que celles qui proviennent de la faillite d'une PME (Bucaille et Costa de Beauregard, 1987). Il faut toutefois également tenir compte du fait que les engagements des établissements financiers dans les grandes firmes représentent des sommes importantes dont le remboursement est liéà la survie de l'emprunteur. Or, il est évident que les répercussions d'une faillite sont largement plus importantes lorsqu'il s'agit d'une grande entreprise, qui fournit du travail de manière directe ou par le biais de la sous-traitance à un grand nombre de salariés, que quand une PME est en jeu. La conjonction de ces deux phénomènes peut ainsi contribuer à expliquer pourquoi l'encadrement et le rationnement du crédit, loin de peser exclusivement sur les firmes les moins performantes, fortement touchées par les crises et financièrement plus vulnérables, a également atteint les PME. Les principales causes de cet état de fait résident en général dans les structures des ressources peu diversifiées de ces deux groupes d'entreprises et dans la dégradation lente, mais continue, de leurs taux d'autofinancement qui incitent les prêteurs à une plus grande concurrence.

\section{Conclusion}

En définitive, il apparaît que les unités de petites et moyennes dimensions se caractérisent au sein du système productif par des comportements et des besoins financiers spécifiques. En effet, si leur endettement global se révèle sensiblement égal à celui des grandes entreprises, une analyse plus fine mettant l'accent sur la structure de l'endettement fait état de la prééminence des crédits à court terme dans les bilans des PME-PMI. La précarité de la situation financière et donc la vulnérabilité de ce groupe d'entreprises se trouvent par ailleurs renforcées par des éléments exogènes trouvant leur origine dans le système bancaire national qui s'est longtemps avéré trop complexe pour ce type d'interlocuteurs. On peut à ce propos également faire état d'une ambiguiité dans le comportement des prêteurs qui n'ont pas contribué à faire disparaître la confusion existant entre le capital de l'entreprise et le patrimoine de son dirigeant. Les réponses apportées à ces problèmes concernent essentiellement les fonds propres et les apports de garanties, le succès rencontré par les prêts participatifs et le développement de la technique du cautionnement mutuel paraissant avoir contribué à l'émergence d'une certaine solidarité entre les banques et les petites unités de production. L'amplification de cette interface s'avère en effet nécessaire à l'insertion des PME dans la concurrence nationale, l'amélioration des mécanismes de financement des opérations à haut risque ainsi que la collaboration sur le plan de la gestion entre les entrepreneurs et les banquiers pouvant être bénéfique, non seulement pour les firmes concernées, mais également pour l'ensemble de l'industrie 
et du secteur tertiaire. Il apparaît en outre que le développement de formes de financement directs et plus particulièrement du capital-risque puisse se présenter comme l'un des éléments de solution au problème du financement des PME. Notons toutefois que la mise en place de modalités visant à favoriser l'accès des unités de petite et moyenne dimensions aux diverses sources de fonds prêtables demeure une priorité nationale et que seule la reconnaissance de leurs spécificités, aussi bien techniques que financières, permettra de résoudre leurs difficultés.

\section{BIBLIOGRAPHIE}

Amar, M., (1987), «Dans l'industrie les P.M.E. résistent mieux que les grandes entreprises», Economie et Statistique, mars, $n^{\circ} 197$, p. 3-11.

Artus, P. et Muet, P. A., (1982), «Politique conjoncturelle et Investissement dans les années 70», Observations et Diagnostics Economiques, juin, $n^{\circ} 1$, p. 61-90.

Baroin, D. et Fracheboud, P., (1983), «Les P.M.E. en Europe et leur contribution à l'emploi», Notes et Etudes Documentaires , $\mathrm{n}^{\circ}$ 4715-4716, Paris, La Documentation Française.

Baumol, W.J., (1982), «Contestable markets, an uprising in the theory of industry structure», American Economic Review, vol. 72, n 1, p 1-15.

Berger, M., (1986), «Segmentation et flexibilité du marché des capitaux externes pour les

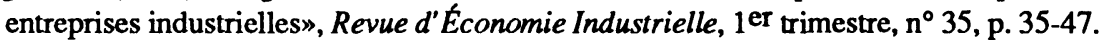

Bernard, J. et Ravix, J.L., (1988), «Diversité et spécificité des unités productives», dans R. Arena, J. de Bandt, L. Benzoni et P.M. Romani (éds), Traité d'Economie Industrielle, Paris, Economica, p. 192-207.

Besse, J., (1983), «P.M.E. et politique industrielle», Revue d'Economie Industrielle, $1 \mathrm{er}$ trimestre, $n^{\circ} 23$, p. 228-243.

Borel, D. et Bouvier, T., (1985), «P.M.E. : la maximisation de l'autofinancement...», Banque, décembre, $n^{\circ} 456$, p. 1144-1148.

Bucaille, A.et Costa de Beauregard, B., (1987),P.M.I.enjeux régionauxet internationaux, Paris, Economica.

Camus, B., (1981), «Rentabilité, investissement et financement», Economie et Statistique, novembre, $n^{\circ} 138$, p. 33-47.

Caves, R. et Porter, M., (1977), «From entry barriers to mobility barriers», Quarterly Journal of Economics, vol. 91, $\mathrm{n}^{\circ} 2$, mai, p. 154-172.

Charreaux, G., (1987), «La théorie positive de l'agence : une synthèse de la littérature», dans C.E.D.A.G., De nouvelles théories pour gérer l'entreprise, Paris, Economica, p. 20-55.

Crédit Hôtelier, (1980), «La petite et moyenne industrie : stratégies de développement», Enquête.

Delattre, M., (1982), «Les P.M.E. face aux grandes entreprises», Economie et Statistiques, octobre, $n^{\circ} 148$, p. 3-19.

Delattre, M., (1986), «Force et faiblesse des secteurs industriels 1979-1984», Collections de l' INSEE, Série $\mathrm{E}, \mathrm{n}^{\circ} 100$, février.

Delbreil, M. et Laudy, J., (1987), «Le financement des P.M.E.», $\mathrm{n}^{\circ}$ spécial des Cahiers Economiques et Monétaires, Banque de France, $n^{\circ} 26$.

Devilliers, M.H., (1987), «P.M.E. ou grandes entreprises, sur qui compter ?», dans J. Dominati (éd) : Quel avenir industriel pour la France?, Paris, Economica.

Flaherty, M.T.,(1980), «Industry structure and cost-reducing investment», Econometrica, juillet, vol. $48, n^{\circ} 5$, p. $1187-1209$. 
Galbraith, J.K., (1959), «Market structure and stabilisation policy», Review of Economics and Statistics, $\mathrm{n}^{\circ} 3$, p. $427-431$.

Galzy, G. (1983), «Le profil de banquier d'entreprise dans les années 1980», Banque, septembre, $n^{\circ} 431$, p. 1145-1151.

Geoffron, P., (1987), «L'État et la dynamique du capital-risque européen», Revue d'Economie Financière, décembre, $n^{\circ} 3$, p. 973-992.

Geoffron, P., (1990), «Le processus de formation d'une innovation financière : le capitalrisque», Thèse pour le Doctorat de Sciences Economiques, Université de Paris XIII, janvier.

Gourlaouen, J.P., (1978), «Rationnement du crédit, risque et pouvoir de marchandage», Revue Economique, $\mathrm{n}^{\circ}$ 5, p. 849-865.

Greffe, X. (éd.), (1984), «Les P.M.E. créent-elles des emplois ?», Paris, Economica.

Guesnier, B., (1986), «Place et évolution des P.M.E. dans le système productif français», Revue d'Economie Régionale et Urbaine, $\mathrm{n}^{\circ}$ 5, p. 609-625.

Gupta, M.C., (1989), «The effect of size, growth and industry on the financial structure of manufacturing companies», Journal of Finance, juin, p. 275-293.

Hourcade, R. et Rosenberg, C., (1980), L'Équilibre financier à court terme de la P.M.E., Paris, Les éditions d'organisation.

Jacquemin, A., (1985), Sélection et pouvoir dans la nouvelle économie industrielle, Paris, Economica.

Julien, P.A. et Morel, P., (1986), La belle entreprise, Montréal, Boréal.

Larrieu, R. et Lombard, N., (1982), «Le comportement économique et financier du secteur secondaire en 1981 », Bulletin Trimestriel de la Banque de France, décembre, ${ }^{\circ} 45$, p.2846.

Larrieu, R. et Lombard, N., (1984), «Le comportement des entreprises du secteur secondaire en 1983», Bulletin Trimestriel de la Banque de France, décembre, n53, p.51-76.

Laudy, J. et Lombard, N., (1984), «Comportement des entreprises en période de «Morsure» de l'encadrement du crédit», Cahiers Économiques et Monétaires, n¹8, p.165-199.

Levratto, N., (1988a), «Les contraintes financières dans les stratégies des firmes», dans R. Arena, J. de Bandt, L. Benzoni et P.M. Romani, Traité d'Économie Industrielle, Paris, Economica, p. 729-742.

Levratto, N., (1988b), «Rationnement ou équilibre du marché du crédit : une analyse fondée sur le comportement des banques», Économies et Sociétés, série monnaie, n 1, p.81-103.

Levratto, N. et Torre, A., (1986), «Logique productive et financement bancaire des entreprises», Revue d'Économie Industrielle, $1^{\circ}$ trimestre, $n^{\circ} 35$, p.195-212.

Marchesnay, M., (1982), «Is Small so Beautiful ?», Revue d'Économie Industrielle, $1^{\circ}$ trimestre, $n^{\circ} 19$.

Mascarenhas, B. et Aaker, D., (1989), «Mobility barriers and strategic groups», Strategic Management Journal, vol 10, p.475-485.

Mayoux, J., (1979), Rapport du groupe de Réflexion sur Le développement des initiatives financières et régionales, annexe $\mathrm{n}^{\circ} 19$, Paris, La Documentation Française.

Mignot, C., (1979), Perspectives de développement de l' artisanat et de lapetiteentreprise, Paris, La Documentation Française.

Mills, D.E. et Schumann, L., (1985), «Industry structure with fluctuating demand», American Economic Review, septembre, vol 75, nº4, p.618-634.

Notes bleues, (1985), Les prêts bonifiés aux entreprises, $\mathrm{n}^{\circ} 232$, Paris, La Documentation Française. 
Pastre, O., (1985), La modernisation des banques françaises, La Documentation Française, novembre.

Portait, R. et Nourel, P., (1982), Les décisions dans l'entreprise, Paris, P.U.F.

Rainelli, M., (1989), Economie industrielle, Paris, Dalloz.

Salvadori, D., (1986), «Le financement des systèmes productifs régionaux», Revue d'Économie Industrielle, $1^{\mathrm{er}}$ trimestre, $\mathrm{n}^{\circ} 35, \mathrm{p} .127-141$.

S.E.S.S.I. (1989), L'État des P.M.I. (éd. 1988), Ministère de l'Industrie et de l'Aménagement du Territoire.

Stigler, G.J.,(1939), «Production and distribution in the shortrun», Journal of Political Economy, juin, vol $47, n^{\circ} 2$.

Stiglitz, J., (1981), «Ownership, control and efficient markets: some paradoxes in the theory of capital markets», dans K.D. Boyer et W.G. Shepherd (éds.) : Economic Regulation: Essays in honor of J.R. Nelsom, M.S.U. Public Utility Papers, p.311-340.

Stiglitz, J., (1985), «Information and economic analysis: a perspective», Economic Journal, Conference Papers, Supplément, vol. 95, p. 137-152.

Vassile, L., (1982), «Les P.M.E. : fragilité financière, forte rentabilité», Economie et Statistique, octobre, $\mathrm{n}^{\circ} 148, \mathrm{p} .21-37$.

Vatteville, E., (1972), Introduction à l'analyse financière de la croissance de la firme, Paris, Cujas.

De Wulf A. et Percie du Sert, P., (1983), «Le Financement des entreprises», dans Préparation $d u I X^{\circ}$ Plan, vol 4, Paris, La Documentation Française. 